\title{
Research and Development of Vibration Control Device Suitable for Detached Houses by Using Displacement Amplification Mechanism*
}

\author{
(Fundamental Characteristic and Vibration \\ Control Performance of Pipe-Type-Damper)
}

\author{
Tomoyoshi WATAKABE**, Satoshi FUJITA***, Toshio OMI***, \\ Hiroshi KURABAYASHI $* * * *$ and Keiji OGATA***** \\ **Graduate School of Advanced Multidisciplinary Engineering, Tokyo Denki University, \\ 2-2 Kanda-Nishiki-cyo, Chiyoda-ku, Tokyo, 101-8457 Japan \\ E-mail:06udq05@ms.dendai.ac.jp \\ *** Department of Mechanical Engineering, Tokyo Denki University \\ 2-2 Kanda-Nishiki-cyo, Chiyoda-ku, Tokyo, 101-8457 Japan \\ **** Vibro-System Co., Ltd., 2-3-18 Sangenjaya, Setagaya-ku, Tokyo, Japan \\ ***** Yacmo Co., Ltd., 5-4-18 Osaki, Shinagawa-ku, Tokyo, Japan
}

\begin{abstract}
The detached houses, in general, have the first natural frequencies in relatively higher range, and their structural deflection is small even though their response acceleration is high. Therefore, the deformation transmitted to damper becomes small, and the dampers have to be designed to obtain a desirable performance against such small amplitude vibrations. For this reason, the new type of damper having the displacement amplification mechanism was developed to solve this problem in this study ("pipe-type-damper"). This paper reports on the results obtained from experiments and analysis of a detached house model using the dampers. In the experiments, the trial damper of $10 \mathrm{kN}$ capacity was made, and the experiments were performed to investigate the fundamental characteristics of the pipe-type-damper. As a result, the properties of the pipe-type-damper were expressed by the fractional derivative 3-element model, its restoring force was proportional to the second power of amplification factor by the amplification mechanism. Analytical results obtained by considering the characteristics of damper showed that the pipe-type-damper had good vibration control performance.
\end{abstract}

Key words: Vibration Control, Amplification Mechanism, Detached Houses, Damper, Viscoelastic Material, Loading Tests, Structural Analysis

\section{Introduction}

In Japan, many types of dampers have been installed into structures such as high-rise buildings, bridges and industrial facilities to reduce excessive responses during earthquakes. In recent years, these dampers begin to be applied to the detached houses having the natural frequency in higher range. However, the detached houses generally produce less structural deflections in various levels even though the response acceleration is very high. Therefore, the deformation transmitted to damper becomes small, and the damper doesn't show a desirable performance against small amplitude vibrations. It is important that not only to minimize the earthquake damage but also to reduce the environmental vibrations to

${ }^{*}$ Received 31 Mar., 2009 (No. T1-07-0812) Japanese Original : Trans. Jpn. Soc. Mech. Eng., Vol.74, No.740, C (2008), pp.930-937 (Received 13 Sep., 2007) [DOI: 10.1299/jsdd.3.368] 
improve the residual comfort, and the dampers should be effective even the small amplitude vibrations. Moreover, the cost of manufacturing dampers should be inexpensive to be widely used. To solve these problems, Noguchi developed the vibration control panel which combined the lever with the bearing wall ${ }^{(1)}$. This panel has been put to practical use in the new detached houses. As shown in this example, to amplify the displacement by applying the principle of leverage, the damper can achieve good performance even if the deformation transmitted to damper is small.

In this study, the new type of damper having the amplification mechanism has been developed ("pipe-type-damper"). Since an amplification mechanism of pipe-type-damper is simple, the damper becomes small-sized. Therefore, this damper can be applied not only to the new detached house but also to the old detached house. This paper describes the results obtained from loading test and analysis of structural model using the pipe-type-damper.

\section{Pipe-Type-Damper}

\subsection{Construction and amplification mechanism}

Picture and schematic drawing of pipe-type-damper is shown in Fig.1 and Fig.2. Figure1 is trial damper used at the loading test. This damper is constructed by shaft, hollow pipe, lever plate, pin, bush, turnbuckle and viscoelastic material. Shaft is inserted in the hollow pipe, and the lever plates are connected by the pin. If the shaft moves, the relative displacement of between the shaft and the hollow pipe is amplified by the lever plates. Amplification mechanism of pipe-type-damper illustrated in Fig.3. Points O,A,B,C and D shown in Fig.3 correspond to each of the points shown in Fig.2. If the displacement $\Delta y$ is given to axial direction of the damper, points A and $\mathrm{B}$ are moved only $\Delta y$, too. Point $\mathrm{C}$ moves on the circular arc as the point $\mathrm{O}$ remains at the center. The displacement of y direction at point $\mathrm{C} \Delta y_{c}$ is calculated by the general solution of simultaneous Eq. (1).

$$
\begin{aligned}
& x_{C}{ }^{2}+y_{C}{ }^{2}=l_{2}{ }^{2} \\
& \left(x_{A}-x_{C}\right)^{2}+\left(\left(y_{A}+\Delta y\right)-y_{C}\right)^{2}=l_{1}^{2}
\end{aligned}
$$

The rotating angle of lever plate $\angle \mathrm{C}^{\prime} \mathrm{OC}=\angle \mathrm{D}^{\prime} \mathrm{OD}=\theta$ becomes as follows.

$$
\theta=\sin ^{-1} \frac{y_{C}}{l_{2}}
$$

From Eq. (2), the length of point O-C $l_{2}$ and the length of point O-D $l_{3}$, the amplification factor $\beta_{x}, \beta_{y}$ of $\mathrm{x}, \mathrm{y}$ direction at point $\mathrm{D}$ against input displacement becomes as follows.

$$
\beta_{x}=\frac{l_{3}(1-\cos \theta)}{\Delta y}, \quad \beta_{y}=\frac{l_{3} \sin \theta}{\Delta y}+1
$$

The displacement of point $\mathrm{B}$ equals input displacement. Therefore, the amplification factor of y direction becomes the value which 1 is added to the amplification factor of lever plate. The amplification factor $\beta$ is finally obtained as follows.

$$
\beta=\sqrt{\beta_{x}^{2}+\beta_{y}^{2}}
$$

\subsection{Construction and Amplification Mechanism}

The restoring force of damper having displacement amplification mechanism is proportional to the second power of the amplification factor as follows.

$$
F_{D}=\beta^{2} K_{v} x+\beta^{2} C_{v} \dot{x}
$$




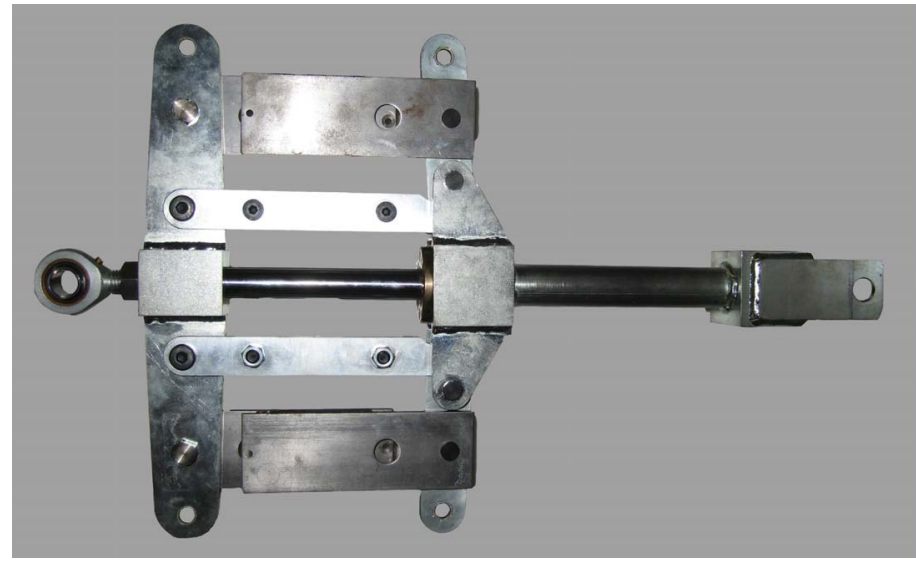

Fig. 1 Trial pipe-type-damper

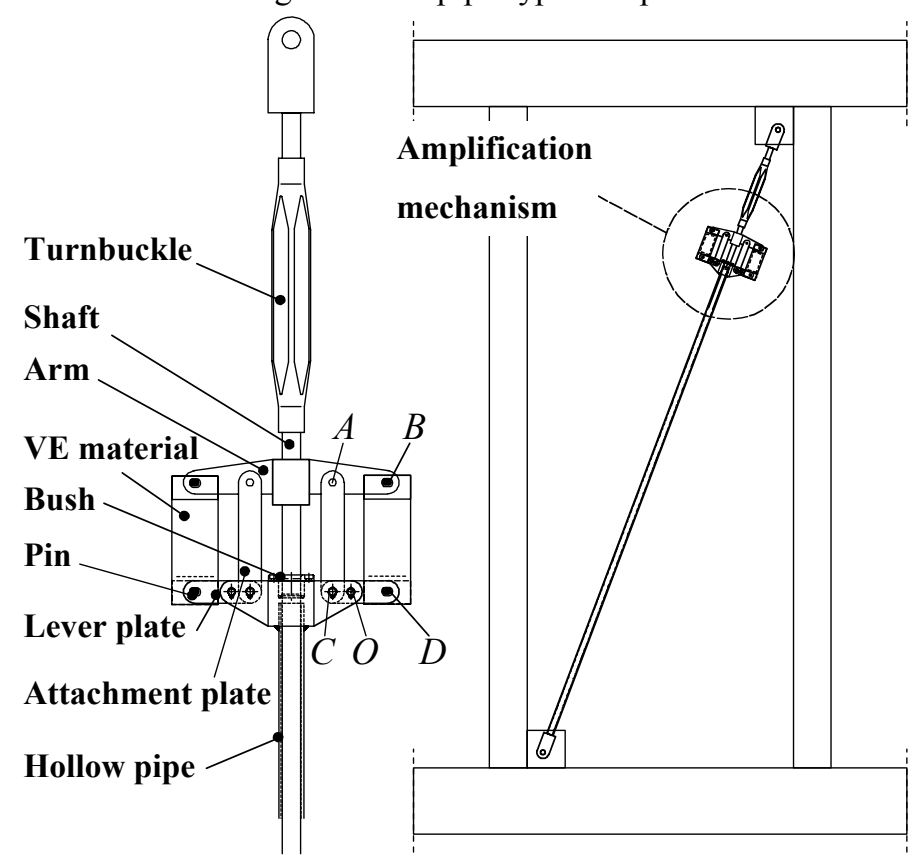

Fig. 2 Schematic drawing of pipe-type-damper

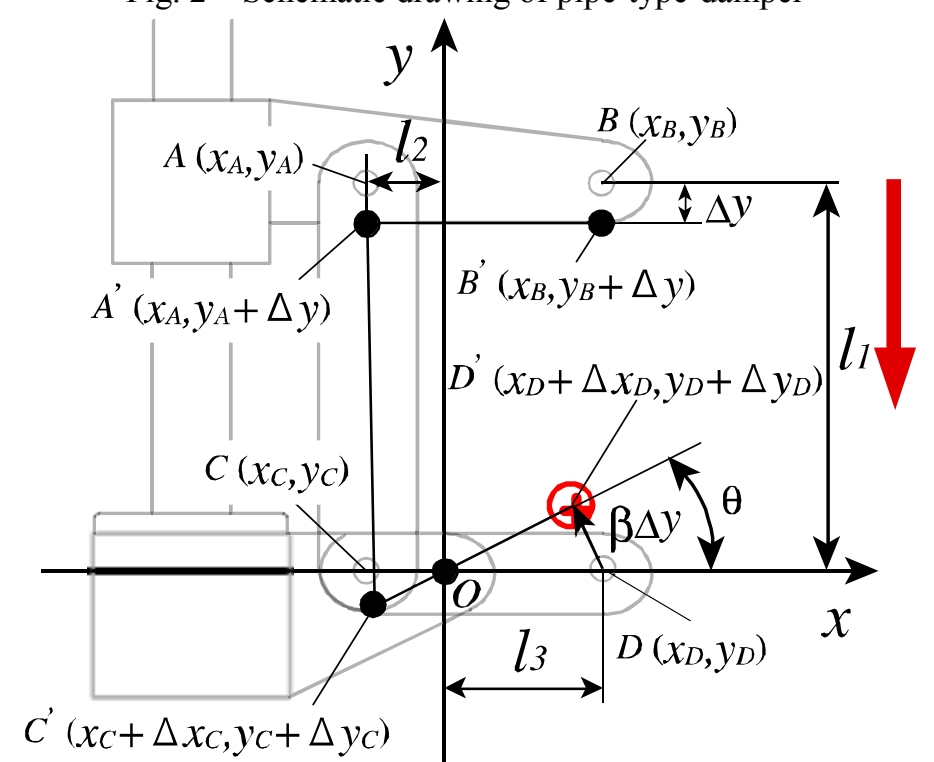

Fig. 3 Amplification mechanism of pipe-type-damper 
where $K_{V}$ is equivalent stiffness of viscoelastic material, $C_{V}$ is equivalent damping coefficient of viscoelastic material. Equivalent stiffness is derived by relationship the shape and the shear force, and equivalent damping coefficient of viscoelastic material is derived by relationship between the shape and the energy which viscoelastic material dissipates in a single cycle. Relation of storage modulus to equivalent stiffness and relation of loss modulus to equivalent damping are as follows.

$$
K_{V}=G^{\prime}\left(\frac{A_{S}}{d}\right), \quad C_{V}=G^{\prime \prime}\left(\frac{A_{S}}{d \omega}\right)
$$

where $d$ is the thickness of viscoelastic material, $A_{S}$ is the area of viscoelastic material. Besides, storage modulus, loss modulus and loss factor are in relation shown in Eq. (7).

$$
\tan \delta=\frac{G^{\prime \prime}}{G^{\prime}}
$$

The viscoelastic material has the non-linear characteristics, and expressing clearly the characteristic value is difficult. Therefore, the modeling shown in the next section is needed to calculate the restoring force of the damper.

\subsection{Characteristics and Modeling of Viscoelastic Material}

The loading test was performed to investigate the characteristic of the viscoelastic material which is used in pipe-type-damper. The used viscoelastic material is the kind of diene polymeric material, and its size is $40 \times 55 \times 10 \mathrm{~mm}$. The input was a sine wave, and the experiment was performed in the following range; frequency: 0.01 to $5 \mathrm{~Hz}$, displacement: 10 to $300 \%$, internal temperature of viscoelastic material: 10 to $40{ }^{\circ} \mathrm{C}$. The characteristics of viscoelastic material were evaluated by storage modulus and loss factor, and hysteresis loop of 3rd. cycle was used to calculate them. From the experiment, it was confirmed that the viscoelastic material had frequency, temperature and displacement dependencies, and these dependencies were modeled as follows.

Frequency and temperature dependencies are modeled by applying frequency-temperature equivalence principle and using fractional derivative 3-element model $^{(2),(3)}$. Figure 4 shows the experimental and analytical results, and experimental data which is obtained in each of frequency and temperature are moved parallel to the horizontal logarithmic axis against reference temperature $20{ }^{\circ} \mathrm{C}$. As shown in Fig. 4, experimental data are on curve line. It has been called frequency-temperature equivalence principle, and this principle is confirmed in the viscoelastic materials which we used, too. The amount of movements in Fig. 4 is shifting factor $\lambda(T)$, and horizontal axis of the graph is defined as equivalence frequency $\lambda(T) f$. Relation of the shifting factor and temperature is plotted in Fig. 5, and the curve approximated by WLF equation (Eq. (8)) is shown at the line.

$$
\lambda(T)=\exp \left(\frac{-C_{1}\left(T-T_{0}\right)}{C_{2}+T-T_{0}}\right)
$$

where $C_{1}$ and $C_{2}$ are coefficient. These value were decided by applying least squares fitting to shifting factor in each temperature, the values were $C_{1}=46, C_{2}=280$. Next, analytical results which is shown at the line in Fig. 5 were calculated by the fractional derivative 3-element model which is shown in Fig. 6, the constitutive equation of this model is as follows.

$$
\sigma+a_{1} D^{\alpha}[\sigma]=b_{1} \gamma+b_{2} D^{\alpha}[\gamma]
$$

where $a_{1}=\eta_{2} / G_{2}, \quad b_{1}=G_{1}, \quad b_{2}=\eta_{2}\left(G_{1}+G_{2}\right) / G_{2}, \quad D^{\alpha}=d^{\alpha} / d t^{\alpha} . a_{1}$ and $b_{2}$ are also expressed to satisfy frequency-temperature equivalence principle: $a_{1}=a_{r} \lambda(T), b_{2}=b_{r} \lambda(T)$. From Eq. (9), storage modulus and loss factor of fractional derivative 3-element model are expressed as follows. 


$$
\begin{aligned}
& G^{\prime}(\omega)=\frac{b_{1}+a_{1} b_{2} \omega^{2 \alpha}+\left(a_{1} b_{1}+b_{2}\right) \omega^{\alpha} \cos \left(\frac{\alpha \pi}{2}\right)}{1+a_{1}^{2} \omega^{2 \alpha}+2 a_{1} \omega^{\alpha} \cos \left(\frac{\alpha \pi}{2}\right)} \\
& \tan \delta(\omega)=\frac{\left(-a_{1} b_{1}+b_{2}\right) \omega^{\alpha} \sin \left(\frac{\alpha \pi}{2}\right)}{b_{1}+a_{1} b_{2} \omega^{2 \alpha}+\left(a_{1} b_{1}+b_{2}\right) \omega^{\alpha} \cos \left(\frac{\alpha \pi}{2}\right)}
\end{aligned}
$$

From interrelation of storage modulus and loss factor of experimental results and Eqs. (10),(11), each parameters were decided as follows: $a_{r}=0.06 \mathrm{~s}^{\alpha}, b_{r}=0.005 \mathrm{Ns} / \mathrm{m}^{2}, b_{l}=$ $6050.91 \mathrm{~N} / \mathrm{m}^{2}, \alpha=0.37$. From Fig.5, it was confirmed that analytical results are good agreement with experimental results. This results indicate the fractional derivative 3 -element model can express frequency and temperature dependencies over the range which experiment was performed; frequency: 0.01 to $5 \mathrm{~Hz}$, internal temperature of viscoelastic material: 10 to $40{ }^{\circ} \mathrm{C}$. Moreover, characteristic of the viscoelastic material can be predicted by this model over the range which experiment wasn't performed; equivalence frequency: 0.004 to $25.52 \mathrm{~Hz}$.

Displacement dependence was expressed by approximated equation of experimental results. Figure 7 shows storage modulus of experimental results plotted against strain (Frequency is $3 \mathrm{~Hz}$, and temperature is $20{ }^{\circ} \mathrm{C}$ ), and all data are normalized by the storage modulus of strain $50 \%$. From experimental results, it was confirmed that storage modulus

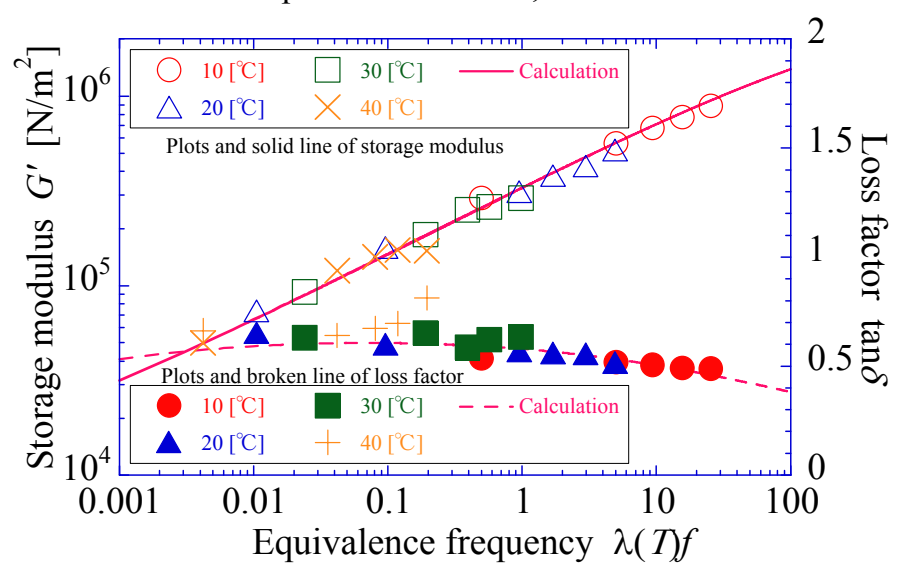

Fig. 4 Results of experiment and analysis against equivalence frequency

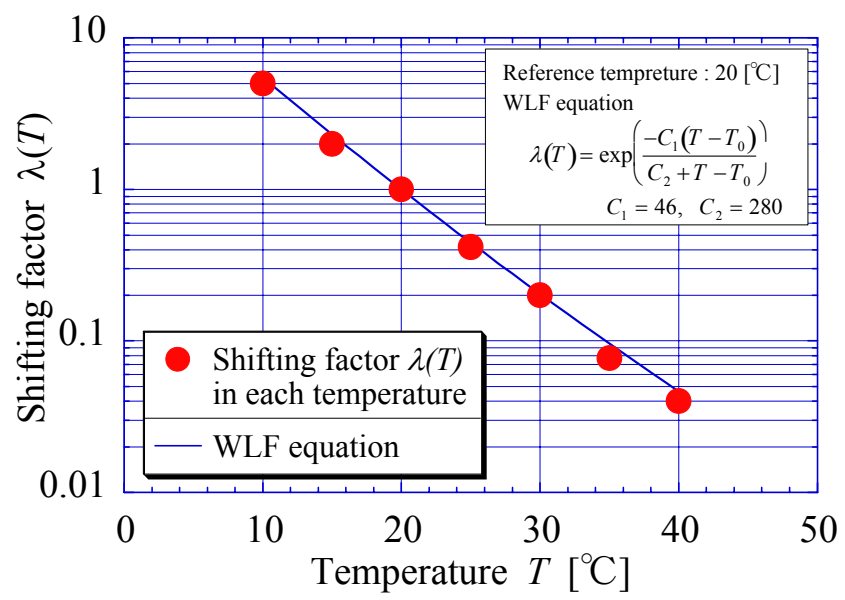

Fig. 5 Relationship of between shifting factor and temperature 
decreased with the increasing strain, loss factor was constant against strain. This results indicate the storage modulus of the used viscoelastic material depends on the strain. Therefore, $b_{1}$ and $b_{2}$ of Eq. (9) were replaced to express displacement dependence of storage modulus by Eq. (12).

$$
\begin{aligned}
& b_{1}=b_{1} A_{G^{\prime}(\gamma)}, \quad b_{2}=b_{r} \lambda(T) A_{G^{\prime}(\gamma)} \\
& A_{G^{\prime}(\gamma)}=1.19-3.00 \times 10^{-3} \gamma+3.86 \times 10^{-6} \gamma^{2}
\end{aligned}
$$

In this time, change rate in less than $20 \%$ was assumed to be constant, and the applied range of Eq. (13) is 10 to $300 \%$. The fractional derivative 3-element model constructed as mentioned above is used as analytical model of viscoelastic material in the next chapter.

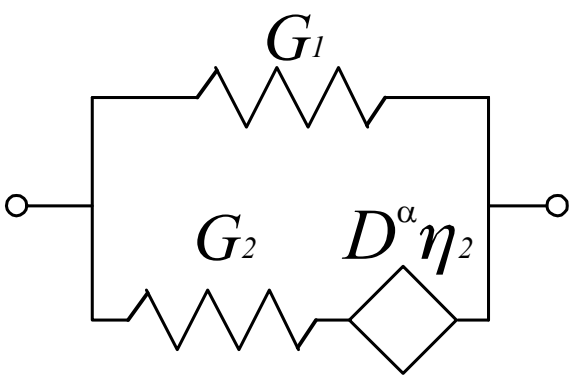

Fig. 6 Fractional derivative 3-element model

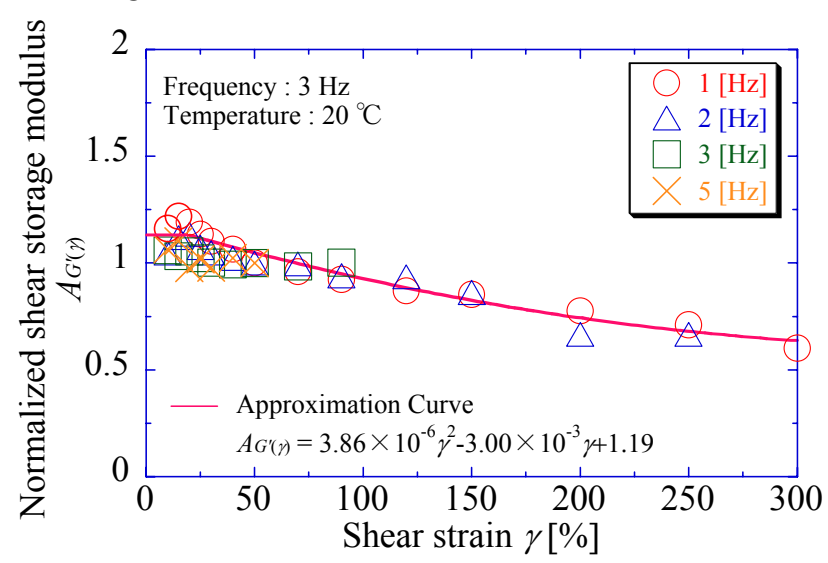

Fig. 7 Storage modulus which was normalized by storage modulus of strain $50 \%$

\section{Loading Test of Pipe-Type-Damper}

\subsection{Experimental Method}

The loading test was performed to investigate the fundamental characteristics of pipe-type-damper. The experimental setup is shown in Fig. 9. Trial-damper shown in Fig. 1 was designed to provide maximum deformation of $\pm 7 \mathrm{~mm}$ and maximum restoring force of $\pm 9.8 \mathrm{kN}$ (In the case of frequency: $3 \mathrm{~Hz}$ and temperature: $20{ }^{\circ} \mathrm{C}$ ). The viscoelastic material which is used in the damper was the same as the viscoelastic material treated in the previous chapter. The actuator used for loading gives a maximum load of $29.4 \mathrm{kN}$ and stroke of $\pm 100 \mathrm{~mm}$. Measurements were made in the restoring force, the input displacement, the deformation of viscoelastic material (amplification displacement) and the temperature (environmental temperature, internal temperature of viscoelastic material). The controlled temperature bath was used in order to regulate temperature, and the inside of the controlled temperature bath was regulated by the air conditioner and the heater. The input was a sine wave, and experiment was performed in the following range; frequency : 1 to $5 \mathrm{~Hz}$, displacement: 1 to $7 \mathrm{~mm}$, internal temperature of viscoelastic material : $20{ }^{\circ} \mathrm{C}$. 
The characteristics of the damper were evaluated by equivalent stiffness and equivalent damping ratio, and these values were defined by Eqs. (14), (15) (Fig. 8).

$$
\begin{gathered}
K_{D}=\frac{F\left(X_{\max }\right)}{X_{\max }} \\
h_{e q}=\frac{1}{4 \pi} \frac{\Delta W}{W}
\end{gathered}
$$

where $X_{\max }$ is maximum displacement, $F\left(X_{\max }\right)$ is force at maximum displacement, $W$ is elastic energy (the area of hatching part), $\Delta W$ is energy which is absorbed at one cycle by the viscoelastic material (the area of loop).

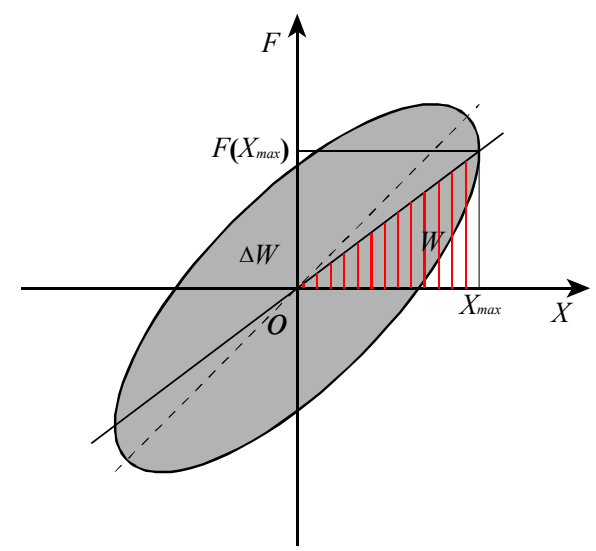

Fig. 8 Calculation method of equivalent stiffness and equivalent damping factor

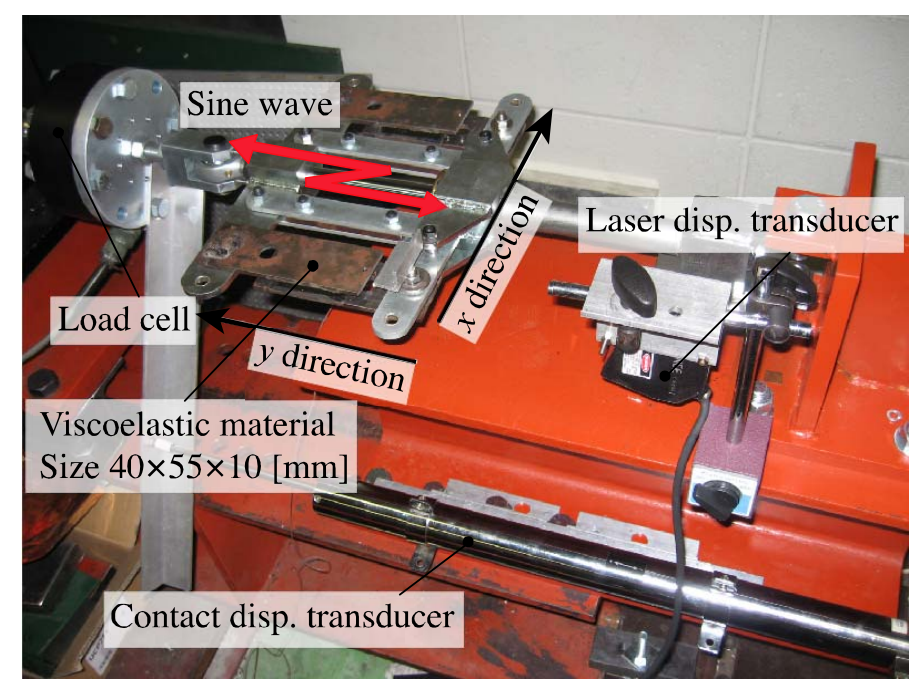

Fig. 9 Schematic drawing of experimental setup

\subsection{Experimental Result}

Figure 10 shows relationship between input displacement and the deformation of viscoelastic material (amplification displacement). The amplification displacement is proportional to input displacement; as a consequence of this, geometric nonlinearity of the amplification mechanism can be neglected. The amplification factor in experiment was slightly smaller than it in theory, and the error ratio was $14 \%$. The discrepancy is due to an insufficient stiffness of lever plate. However, considering the error factor, theoretical value which is calculated by Eq. (5) is agreement with experimental result. Hence, the validity of theoretical equation was demonstrated through the loading test.

Figure 11 shows hysteresis loops of experiment and analysis at each displacement and 
frequency. Analytical results were calculated by substituting the fractional derivative 3-element model constructed at previous chapter for Eq. (5). In this graph, it is confirmed that experimental qualitative result agrees with analytical qualitative result. This result indicates that restoring force of pipe-type-damper is proportional to the second power of amplification factor by the amplification mechanism. Equivalent damping ratio of damper was $23 \%$ in experiment and $25 \%$ in analysis in the case of frequency of $3 \mathrm{~Hz}$ and displacement of $7 \mathrm{~mm}$, and the error ratio was about $10 \%$. This error factor is due to a decrease of experimental hysteresis loop's area that occurs in backlash of pin.

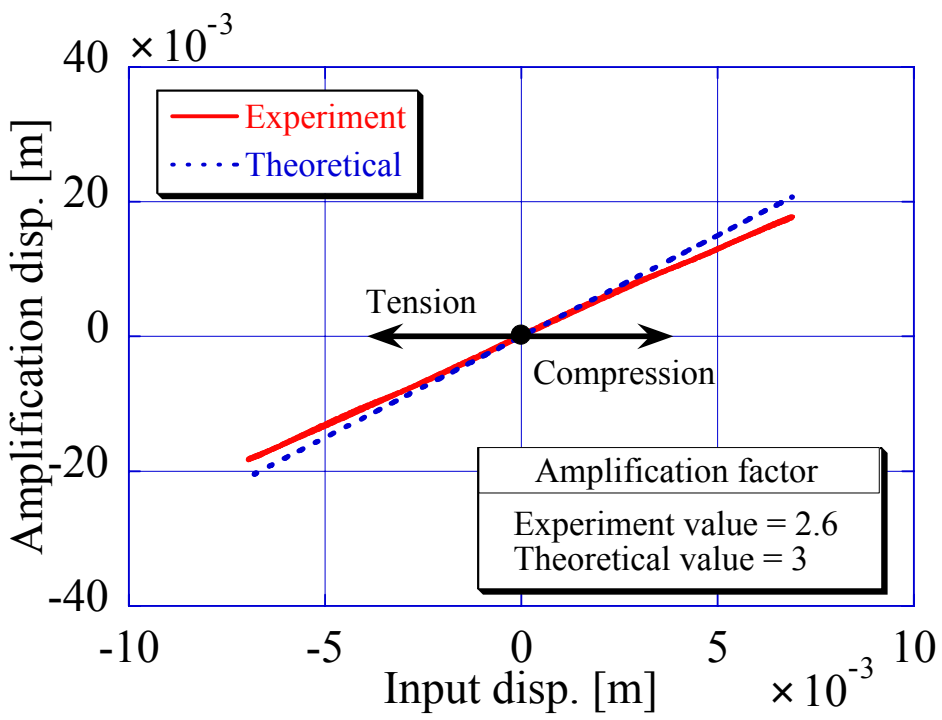

Fig. 10 Comparison of amplification displacement and input displacement
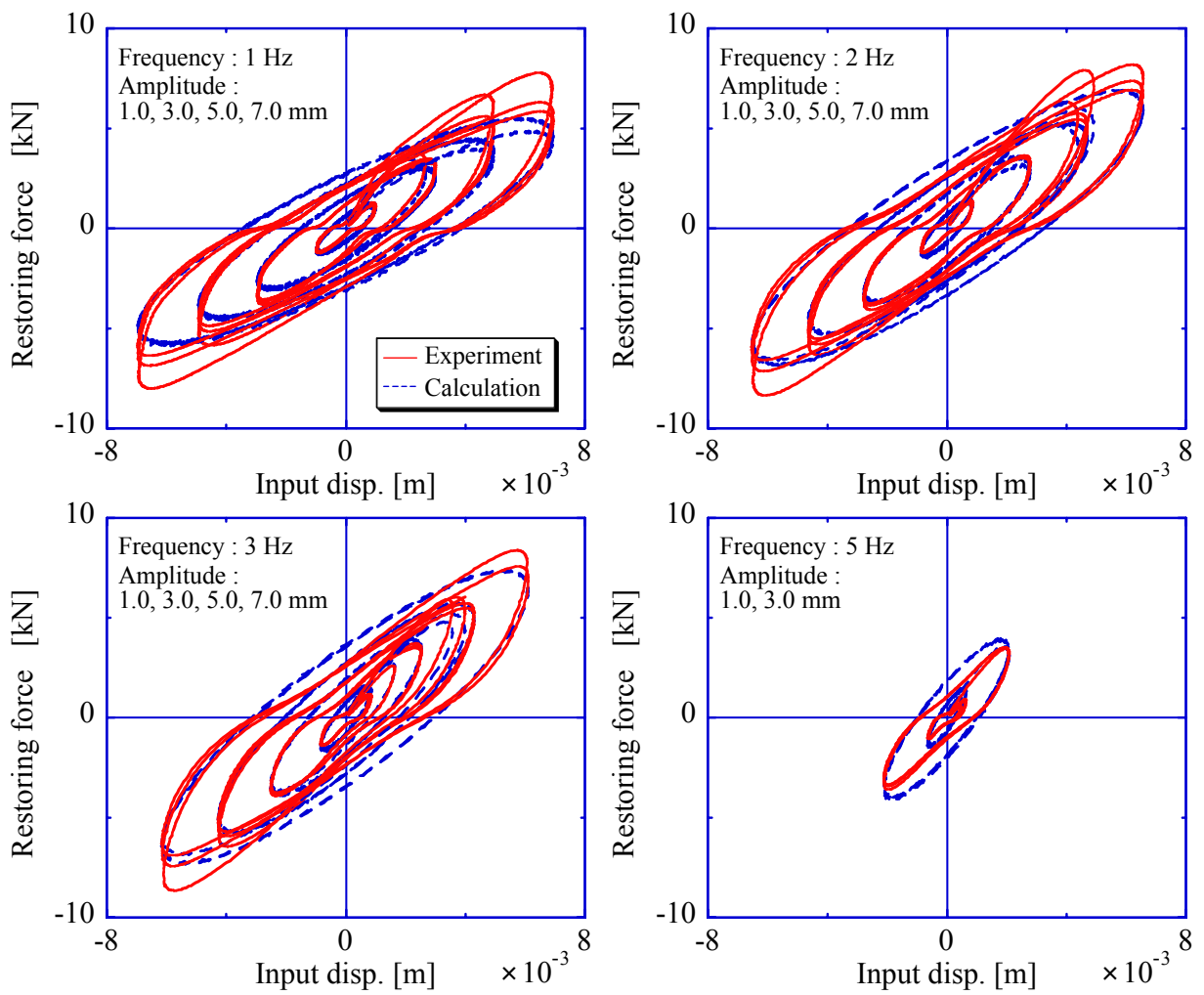

Fig. 11 Hysteresis loop characteristics of pipe-type-damper 


\section{Loading Test of Pipe-Type Damper}

\subsection{Analytical Method}

Seismic response analysis was performed to investigate the performance of pipe-type-damper. Analytical model is two degree of freedom non-linear system as shown in Fig.12. Bi-linear+Slip model shown in Fig. 13 was used as restoring force characteristic of the structure, and the parameter of structural model are shown in Table 1 and Table $2^{(4)}$. From the result of eigenvalue analysis, the first natural frequency of the structural model is $3.40 \mathrm{~Hz}$, and the second natural frequency is $9.90 \mathrm{~Hz}$. The analytical model of pipe-type-damper was fractional derivative 3-element model, and the result of loading test was reflected in analytical model of damper. Influence of supporting part of the damper was also considered in the analysis, the value was $2.05 \times 10^{8} \mathrm{~N} / \mathrm{m}$. The damper was obliquely installed in the structure, and it was only installed in first story. Equations of motion in the analysis are as follows.

$$
\begin{aligned}
& m_{1} \ddot{x}_{1}+c_{1} \dot{x}_{1}-c_{2} \cdot\left(\dot{x}_{2}-\dot{x}_{1}\right)+F_{B S 1}(x)-F_{B S 2}(x)+F_{D H 1}(x)=-m_{1} \ddot{z}_{H} \\
& m_{2} \ddot{x}_{2}+c_{2} \cdot\left(\dot{x}_{2}-\dot{x}_{1}\right)+F_{B S 2}(x)=-m_{2} \ddot{z}_{H}
\end{aligned}
$$

where $m_{1}$ and $m_{2}$ are mass at each story, $c_{1}$ and $c_{2}$ are damping coefficient at each story of the structure, $F_{B S I}(x)$ and $F_{B S 2}(x)$ are the restoring force at each floor of the structure, $F_{D H 1}$ and $F_{D H_{2}}$ are the restoring force of the damper at each floor, $\ddot{z}_{H}$ is horizontal acceleration at the ground.

There are the following three cases as the analytical cases in this analysis.

Case 1 is: Damper isn't installed in the structural model.

Case 2 is: Damper without amplification mechanism is installed in the structural model, and the added damping ratio is $0.9 \%$ against first natural frequency of structural model. (Non-amplification mechanism damper).

Case 3 is: Pipe-type-damper is installed in the structural model, and the added damping ratio is $6 \%$ against first natural frequency of structural model.

Table 1 Parameter of analytical model

\begin{tabular}{ccc}
\hline Mass2 & 16900 & {$[\mathrm{~kg}]$} \\
\hline Mass1 & 12100 & {$[\mathrm{~m}]$} \\
\hline Height of floor & 2.73 & {$[\%]$} \\
\hline Damping ratio of 1st. mode & 5 & \\
\hline
\end{tabular}

\begin{tabular}{|c|c|c|c|c|c|c|}
\hline \multirow{5}{*}{$\begin{array}{l}\text { Bi-linear } \\
\text { element }\end{array}$} & \multirow{2}{*}{ 1st. story } & 1st. stiffness & $k_{b 1}$ & 7.89 & 1.86 & \multirow{4}{*}[\mathrm{kN}/\mathrm{m}]{} \\
\hline & & 2nd. stiffness & $k_{b 2}$ & 0.45 & 0.06 & \\
\hline & \multirow{2}{*}{ 2nd. story } & 1st. stiffness & $k_{b 1}$ & 8.73 & 1.99 & \\
\hline & & 2nd. stiffness & $k_{b 2}$ & 0.49 & 0.07 & \\
\hline & \multicolumn{2}{|c|}{ 1st. yield disp. } & $x_{1}$ & 4.5 & 2.25 & $\times 10^{-3}[\mathrm{~m}]$ \\
\hline \multirow{8}{*}{$\begin{array}{l}\text { Slip } \\
\text { element }\end{array}$} & \multirow{3}{*}{ 1st. story } & 1st. stiffness & $k_{s 1}$ & 6.98 & 1.40 & \multirow{6}{*}[\mathrm{kN}/\mathrm{m}]{} \\
\hline & & 2nd. stiffness & $k_{s 2}$ & 1.05 & 0.06 & \\
\hline & & 3rd. stiffness & $k_{s 3}$ & 1.62 & -0.16 & \\
\hline & \multirow{3}{*}{ 2nd. story } & 1st. stiffness & $k_{s 1}$ & 7.72 & 1.50 & \\
\hline & & 2nd. stiffness & $k_{s 2}$ & 1.17 & 0.07 & \\
\hline & & 3rd. stiffness & $k_{s 3}$ & 1.80 & -0.17 & \\
\hline & \multicolumn{2}{|c|}{ 1st. yield disp. } & $x_{2}$ & 18 & 9 & \multirow{2}{*}{$\times 10^{-3}[\mathrm{~m}]$} \\
\hline & \multicolumn{2}{|c|}{ 2nd yield disp. } & $x_{3}$ & 90 & 45 & \\
\hline
\end{tabular}

Table 2 Parameter of bilinear-slip model

Non- Hatching : Structural plywood and siding board, Hatching : Plaster board 


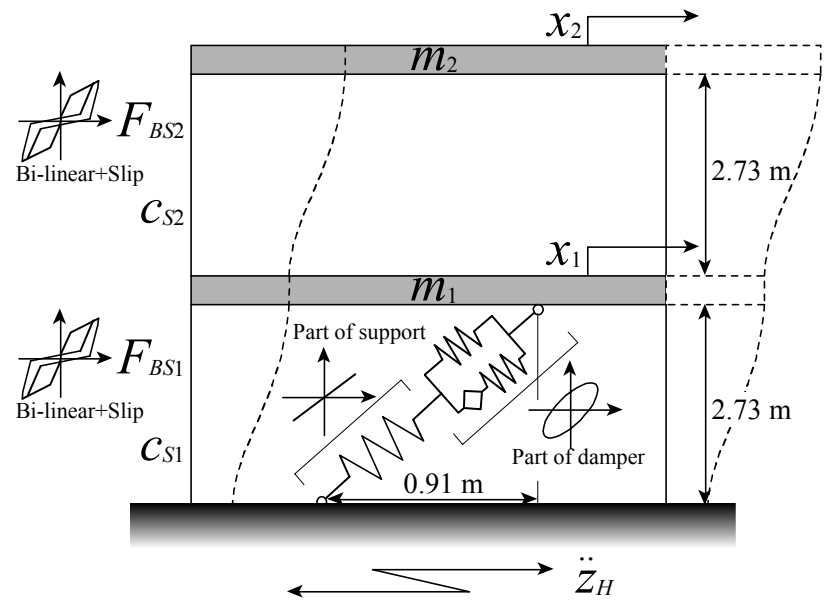

Fig. 12 Analytical model

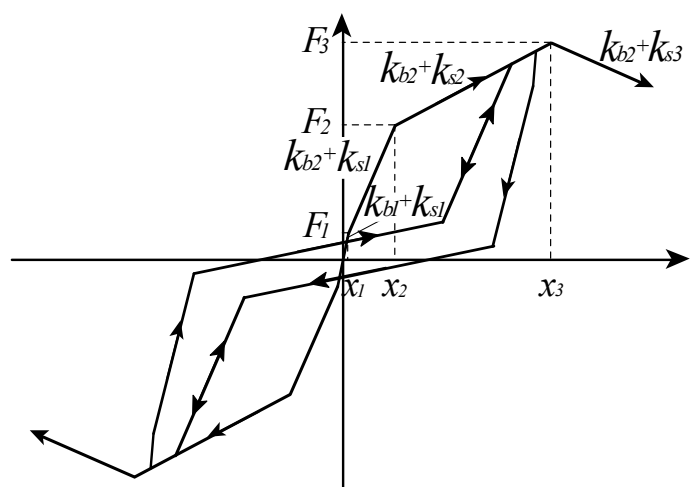

Fig. 13 Bi-linear + Slip model

Difference of non-amplification mechanism damper (Case 2) and pipe-type-damper (Case 3) is only amplification factor $\beta$ : $\beta$ of non-amplification mechanism damper is $1, \beta$ of pipe-type-damper is 2.6 (experimental result of loading test). The used earthquakes were JMA Kobe 1995 NS, BCJ-L2 (An artificial earthquake wave: Building Center of Japan) and traffic vibration (The data is the constant random wave which is observed near the expressway in Tokyo when the car running. Though this wave doesn't break the detached house, this wave gives the habitant the uncomfortable feeling.). The peaks of velocity at and BCJ-L2 are normalized $0.50 \mathrm{~m} / \mathrm{s}$, and JMA Kobe NS and traffic vibration are original wave (Maximum acceleration of JMA Kobe NS is $8.19 \mathrm{~m} / \mathrm{s}^{2}$, and Maximum acceleration of traffic vibration is $2.01 \times 10^{-2} \mathrm{~m} / \mathrm{s}^{2}$ ).

\subsection{Analytical Result}

Figure 14 shows a comparison between the maximum response acceleration and the relative displacement in each floor. From a comparison between Case 1 and Case 2, following things were confirmed: Pipe-type-damper has not excessively increased the maximum response acceleration of structure, and the maximum acceleration in first story were reduced into 90 to $74 \%$ except JMA Kobe NS. The response relative displacement in first story was reduced into 76 to $47 \%$ in all waves. The maximum acceleration was increased by installation of pipe-type-damper in JMA Kobe NS. This is due to prevent the plastic deformation of structure by pipe-type-damper. However, reduction performance of the response relative displacement was the highest in all waves.

From a comparison between Case 2 and Case 3, it is confirmed that pipe-type-damper reduced the response acceleration and the response relative displacement in first story 

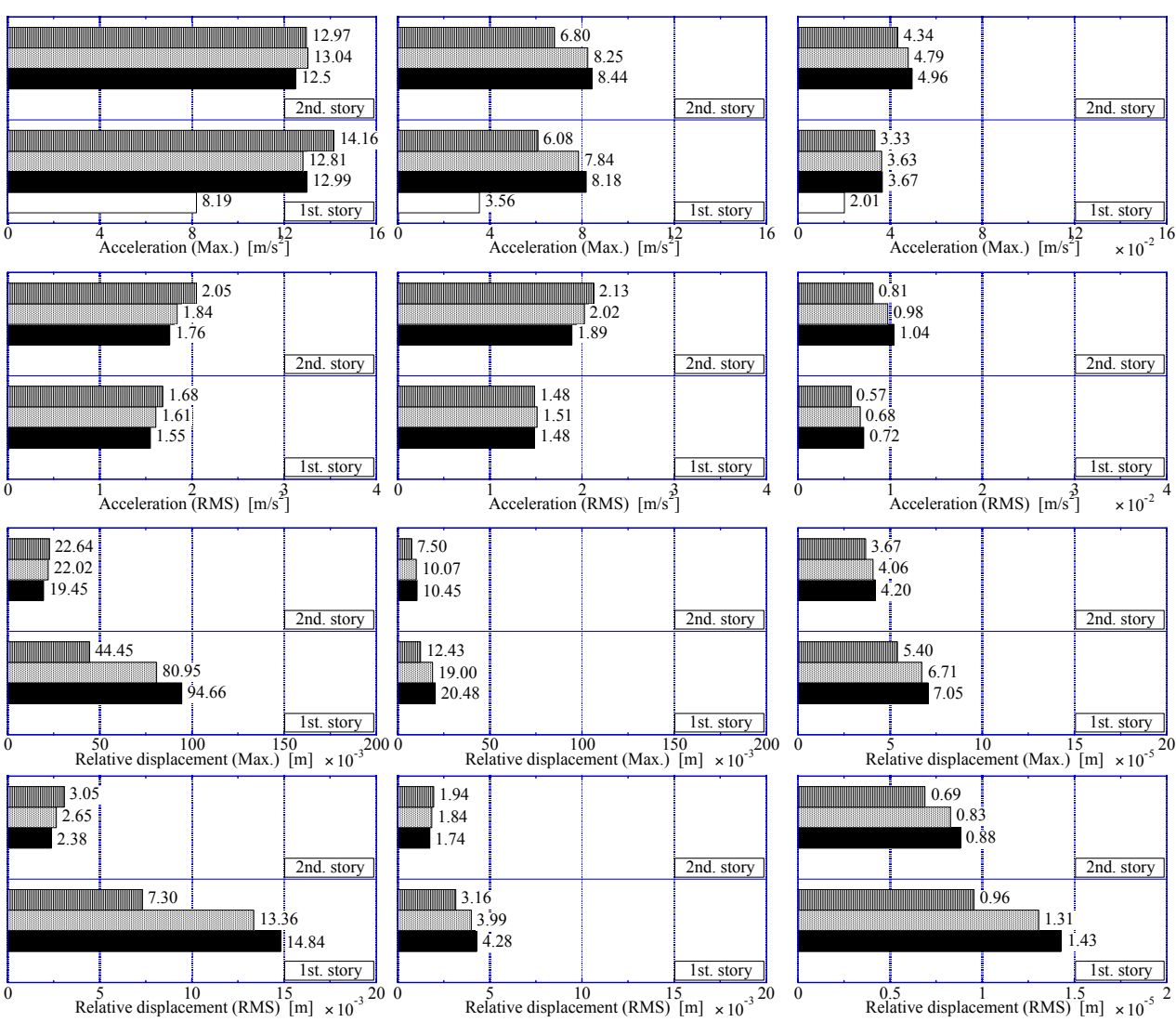
JMA Kobe 1995 NS

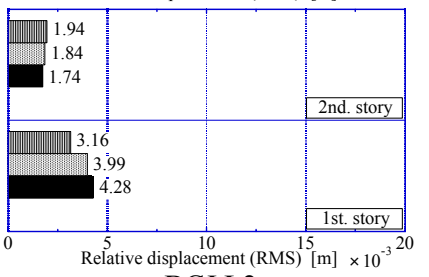

BCJ L2

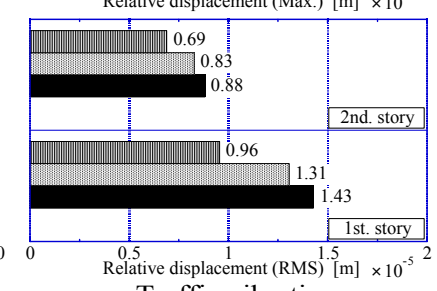

Traffic vibration

$\square$ Input $\square$ Without damper

With non-amp. mech. damper

With pipe-type-damper

Fig. 14 Response acceleration and relative displacement in each floor due to Kobe NS, BCJ L2 and traffic vibration

compared with the responses of structure with non-amplification-mechanism-damper. In other words, reduction of damping material and miniaturization of damper can be realized by using amplification mechanism. From above results, good vibration control performance of pipe-type-damper was confirmed.

\section{Conclusion}

New vibration control device, the pipe-type-damper was described in this report. The knowledge gained by this study is concluded as follows.

(1) The viscoelastic material used with pipe-type-damper has frequency, temperature and displacement dependencies. The characteristics can be simulated by the fractional derivative 3-element model over the following range; frequency: 0.01 to $5 \mathrm{~Hz}$, internal temperature of viscoelastic material: 10 to $40{ }^{\circ} \mathrm{C}$, strain: 10 to $300 \%$.

(2) Geometric nonlinearity of the proposed amplification mechanism can be neglected, and the restoring force of pipe-type-damper was proportional to the second power of amplification factor by the amplification mechanism.

(3) Pipe-type-damper reduced the response acceleration of structure into about 3/4 in the maximum compared with the response structure without damper, and it reduced the response relative displacement into about $1 / 2$ in the maximum.

In this report, amplification mechanism and basically characteristics of pipe-type-damper was examined. Based on these results, we plan to implement the dynamic vibration tests in the future to examine the vibration control performance against earthquake and traffic vibration. 


\section{Acknowledgement}

The authors would like to gratefully acknowledge the students of the Tokyo Denki University for help with the experiment.

\section{References}

(1) Hirata, T., Nishizawa, T., Kajikawa, H., Yoshida S., Chihara K., Mitsuhashi, A., Matsumoto, T. and Noguchi, H., A study on response control structure by wooden panel construction part1-7, Summaries of Technical Papers of Annual Meeting Architectural Institute of Japan, Vol. II (2005), pp. 61-76.

(2) Suzuki, K., Viscoerastic Damping Technology Handbook, (2003), pp. 54-56, 73, Maruzen Co., Ltd.

(3) Kasai, K., Teramoto, M., Okuma, K. and Tokoro, K., Constitutive Rule for Viscoelastic Materials Considering Temperature, Frequency, and Strain Sensitivities, Journal of the Japan Structure of Construction Engineers, Vol. 543 (2001), pp. 77-86.

(4) Iiba, M., Abe A., Hanai T. and Minagawa T., Performance Verification of Low-cost Compact Seismometer and Its Application to Earthquake Motion Observation of Detached House, Journal of the Japan Association for Earthquake Engineering, No. 7 (2007), pp. 57-67. 